\title{
Estimation of pion-emitting source in symmetric and asymmetric collisions using the UrQMD model
}

\author{
Eugenia Khyzhniak $^{1, *}$, Varvara Semenova ${ }^{1}$, Nikita Ermakov ${ }^{1}$, and Grigory Nigmatkulov ${ }^{1}$ \\ ${ }^{1}$ National Research Nuclear University MEPhI, Kashirskoe shosse, 31, 115409 Moscow, Russia
}

\begin{abstract}
The femtoscopy technique allows one to measure spatial and temporal characteristics of the particle-emitting source produced in high-energy collisions. In non-central ultrarelativistic heavy-ion collisions the emission source can be tilted in the reaction plane. In the current analysis, the orientation of freeze-out distributions with respect to the first-order event plane in symmetric $(\mathrm{Au}+\mathrm{Au})$ and asymmetric $(\mathrm{Cu}+\mathrm{Au})$ collisions at $\sqrt{s_{N N}}=200 \mathrm{GeV}$ was studied using the UrQMD model to extract information about the emission source tilt. The effect of the initial geometry on the femtoscopic radii of small systems was measured at the same number of charged particles and transverse momentum was studied in $\mathrm{d}+\mathrm{Au}$ and ${ }^{3} \mathrm{He}+\mathrm{Au}$ collisions at $\sqrt{s_{N N}}=200 \mathrm{GeV}$. The implications of the results are discussed.
\end{abstract}

\section{Introduction}

The femtoscopy technique allows one to measure the spatial and temporal characteristics of the emitting region in high-energy collisions. The femtoscopic correlations arise from the quantum statistics, Coulomb and strong final-state interactions [1]. In order to perform realistic estimations of these effects, the Ultrarelativistic Quantum Molecular Dynamics model (UrQMD 3.4) [2-5] was used in this work. The UrQMD is a microscopic Monte Carlo model for ultrarelativistic ion-ion collision simulation in the energy range from Bevalac and SIS up to AGS, SPS and RHIC. The model is aimed at providing simulations of: the production of dense hadronic matter at high temperatures, mesonic matter and of anti-matter; production and transport of rare particles in hadronic matter; production, modification and destruction of strangeness in matter; emission of electromagnetic probes.

To investigate the effect of the initial overlap geometry on the femtoscopic radii, the femtoscopy of small systems $\left(\mathrm{d}+\mathrm{Au}\right.$ and $\left.{ }^{3} \mathrm{He}+\mathrm{Au}\right)$ at the same number of charged particles and transverse momentum were estimated. The emission source tilt $[6,7]$ and initial conditions influence on it were studied in $\mathrm{Cu}+\mathrm{Au}$ and $\mathrm{Au}+\mathrm{Au}$ collisions at $\sqrt{s_{N N}}=200 \mathrm{GeV}$.

\section{Femtoscopy}

The femtoscopic correlations are calculated as a function of relative momentum, expressed as $q=p_{1}-p_{2}$, where $p_{1}$ and $p_{2}$ are particle four-momenta. In order to estimate the particleemitting source parameters, one uses the correlation function, $C(q)$. It can be written as:

$$
C(q)=\frac{A(q)}{B(q)}
$$

\footnotetext{
*e-mail: eugenia.sh.el@gmail.com
} 
where $A(q)$ is a weighted distribution of the two-particle relative momentum that contains quantum statistical correlations, and $B(q)$ is the same relative momentum distribution obtained from Monte Carlo generator directly. In this work, only Bose-Einstein statistics of two spinless bosons was taken into account in the weight calculation: weight $=1+\cos (q \cdot \Delta x)$, where $\Delta x=x_{1}-x_{2}$ is the difference of particle four-coordinates.

Following the modern analysis techniques, we decompose the relative momentum of the pairs into the three projections, namely out, side and long, according to the Bertsch-Pratt parameterization $[8,9]$. Correlation functions were constructed in the longitudinally co-moving system (LCMS), where $p_{z, 1}+p_{z, 2}=0$. Assuming a Gaussian emission profile of the source, the correlations functions were fitted with the following function $[10,11]$ :

$$
C(q)=1+\lambda \exp \left[-\sum_{i, j=\text { out }, \text { side }, \text { long }} q_{i} q_{j} R_{i j}^{2}\right],
$$

where $\lambda$ is the fraction of correlated pairs and $q_{i}$ is the relative momentum of the pair in the $i$ direction. The out, side and long correspond to the Bertsch-Pratt parameterization. The longitudinal direction along the beam axis corresponds to the long term, the outward direction is pointing along the transverse component of the average momentum $\mathbf{k}$ of a pair $\left(k_{T}=\left|\mathbf{p}_{\mathbf{1}, \mathbf{T}}+\mathbf{p}_{\mathbf{2}, \mathbf{T}}\right| / 2\right)$ and the sideward direction is orthogonal to the both - out and long. The femtoscopic radii are related to homogeneity regions. According to [12], $R_{\text {side }}$ carries information about geometry, $R_{\text {out }}$ convolutes the information about geometry and emission duration and $R_{\text {long }}$ contains information about the system lifetime. The $k_{T}$ dependence of the femtoscopic radii is sensitive to the dynamics of the system evolution and allows one to probe different regions of the homogeneity [13].

For the one dimensional analysis we used two different widely used approximations for the source function: Lorentzian and Gaussian. The one-dimensional correlation functions for these two approximations are:

$$
\begin{aligned}
& \text { Lorentzian: } C\left(q_{i n v}\right)=1+\lambda e^{-R_{i n v} q_{i n v}} \\
& \text { Gaussian: } C\left(q_{i n v}\right)=1+\lambda e^{-R_{i n}^{2} q_{i n v}^{2},}
\end{aligned}
$$

where $q_{\text {inv }}=\sqrt{\left(\overrightarrow{p_{1}}-\overrightarrow{p_{2}}\right)^{2}-\left(E_{1}-E_{2}\right)^{2}}$ - relative four-momentum of the pair; $\lambda$ - correlation strength.

\section{One-dimensional femtoscopy}

To study the dependence of the pion-emitting source size on the transverse momentum of the pion pairs and multiplicity of events, the one-dimensional correlation functions were calculated in the UrQMD model for $\mathrm{d}+\mathrm{Au}$ and ${ }^{3} \mathrm{He}+\mathrm{Au}$ collisions at $\sqrt{s_{N N}}=200 \mathrm{GeV}$. The final-state charged particle multiplicity, $N_{c h}$, was used to constrain the initial geometry of the collision region [14]. The $N_{c h}$ was calculated for the particles in the pseudorapidity range $|\eta|<0.5$ and with $p_{T}>0.15 \mathrm{GeV} / \mathrm{c}$, which correspond to the acceptance of the STAR experiment. Pions with $0.15<p_{T}(\mathrm{GeV} / \mathrm{c})<1.45$ and $|\eta|<1$ were used in this study. Femtoscopic analysis was performed for 8 regions of multiplicity $0 \leq N_{c h} \leq 4,5 \leq N_{c h} \leq 9,10 \leq N_{c h} \leq 14$, $15 \leq N_{c h} \leq 19,20 \leq N_{c h} \leq 24,25 \leq N_{c h} \leq 29$ and $30 \leq N_{c h} \leq 34$. For each multiplicity interval the correlation functions were studied for 7 regions of pair transverse momentum, $k_{T}=0.5\left(p_{1, T}+p_{2, T}\right): 0.15-0.25,0.25-0.35,0.35-0.45,0.45-0.55,0.55-0.65,0.65-$ 0.75 and $0.75-0.85 \mathrm{GeV} / \mathrm{c}$. Thus, multiplicity selections provide constraints on the initial geometry of the collision while measuring $k_{T}$ dependence reflects the dynamics of the system evolution. 

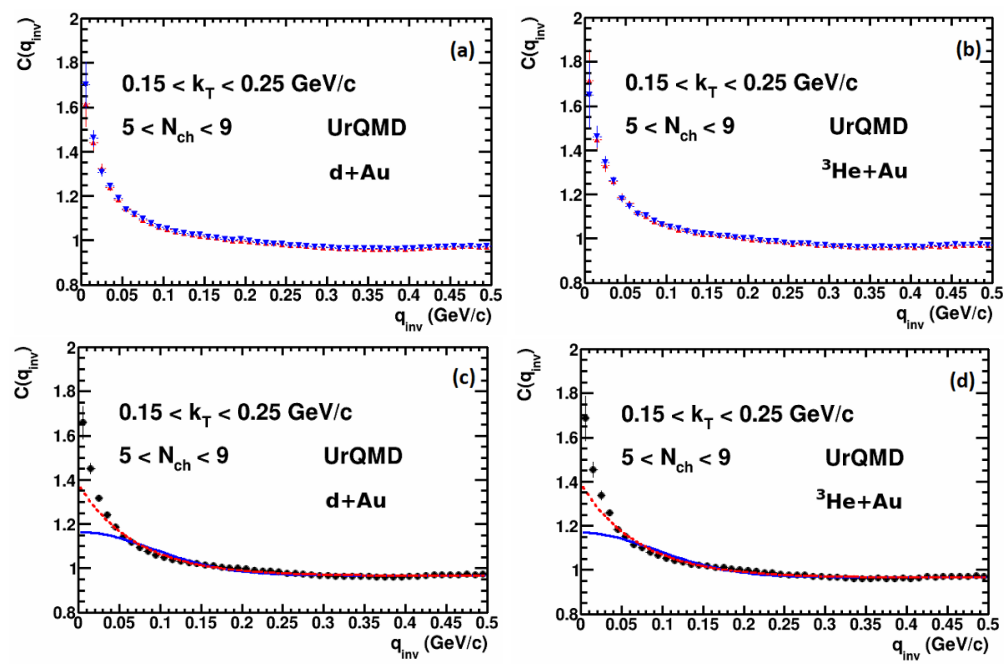

Figure 1. (Left and right panels show correlation functions for $5<N_{c h}<9$ and $0.15<k_{T}(\mathrm{GeV} / \mathrm{c})<0.25$ obtained for $\mathrm{d}+\mathrm{Au}$ and ${ }^{3} \mathrm{He}+\mathrm{Au}$ collisions at $\sqrt{s_{N N}}=200 \mathrm{GeV}$, respectively. (Top panel) Comparison of the correlation functions measured for positive (red triangles) and negative (blue triangles) pion pairs. (Bottom panel) Correlation functions of the combined positive and negative pairs. Blue solid and red dashed lines represent fits by Eqs. 3a and 3b
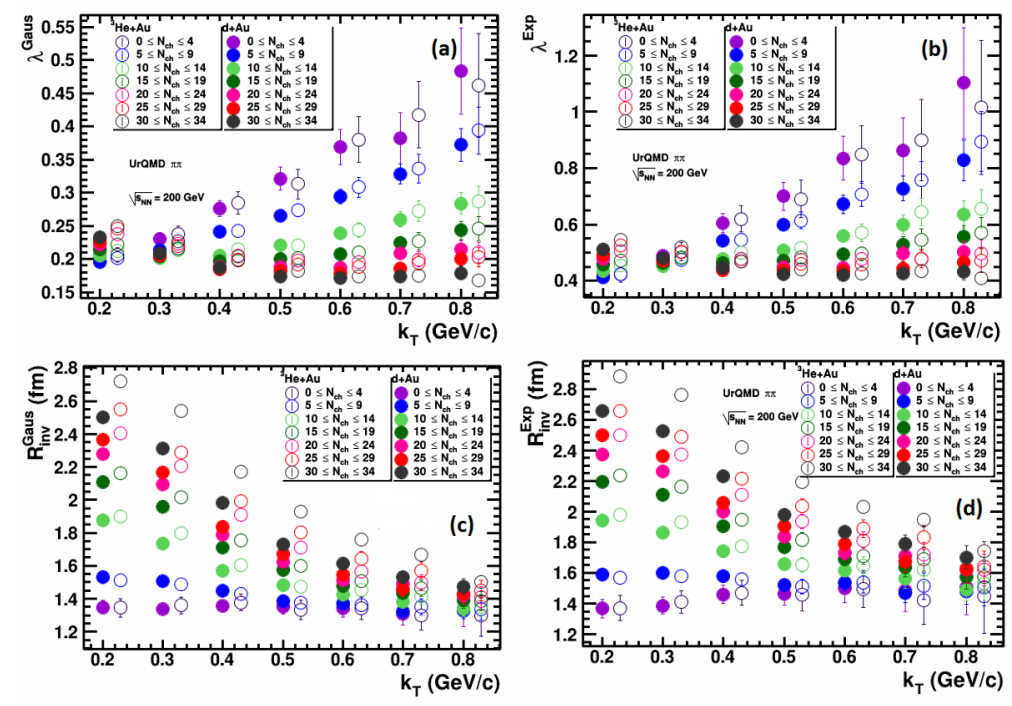

Figure 2. The dependence of the extracted $\lambda$ (top panel) and $R_{i n v}$ (bottom panel) on $N_{c h}$ and $k_{T}$ for $\mathrm{d}+\mathrm{Au}$ collision systems (solid symbols) and ${ }^{3} \mathrm{He}+\mathrm{Au}$ (open symbols) at $\sqrt{s_{N N}}=200 \mathrm{GeV}$. Left and right panels correspond to the results obtained under Gaussian and Lorentzian source assumptions, respectively 
Figs. $1 \mathrm{a}$ and $1 \mathrm{~b}$ show the correlation functions of positive and negative pion pairs. Since the correlation functions are consistent within uncertainties, the relative momenta of positive and negative pairs (numerators and denominators separately) were combined before constructing the correlation functions. The fits to the correlation functions were performed using Eq. 3a (Lorentzian source) and Eq. 3b (Gaussian source) and shown in Figs. 1c and 1d as red dashed and blue solid lines, respectively. The exponential form describes the correlation function better as compared to the Gaussian one.

Fig. 2 shows the extracted $N_{c h}$ and $k_{T}$ dependence of $\lambda$ (top row) and $R_{i n v}$ (bottom row) for $\mathrm{d}+\mathrm{Au}$ (solid markers) and ${ }^{3} \mathrm{He}+\mathrm{Au}$ (open markers). The dependence for the Gaussian and Lorentzian source shapes are shown on the left- and right-hand sides in Fig. 2, respectively. From the comparison one can see that the radii obtained using exponential fits are generally larger than those for Gaussian fits and the difference increases with increasing $N_{c h}$.

The correlation strength extracted from the exponential is about a factor of 2 larger than that for the Gaussian fits for the same $N_{c h}$ and $k_{T}$. The decrease of the radii for the larger $N_{c h}$ with increasing $k_{T}$ reflects the influence of the radial flow. The $R_{i n v}$ obtained for $\mathrm{d}+\mathrm{Au}$ and ${ }^{3} \mathrm{He}+\mathrm{Au}$ collisions at $\sqrt{s_{N N}}=200 \mathrm{GeV}$ are consistent with each other for $N_{c h}<20$. For $N_{c h}>20 R_{i n v}$ measured for ${ }^{3} \mathrm{He}+\mathrm{Au}$ collisions are larger than that for $\mathrm{d}+\mathrm{Au}$ for the same $N_{c h}$ and $k_{T}$. This measurement demonstrates that the initial geometry of different collision systems cannot be constrained by selecting similar $N_{c h}$.

\section{Azimuthally sensitive three-dimensional femtoscopy}

In heavy-ion collisions the particle-emitting source can be tilted in the reaction plane. In order to theoretical study the effect and its dependence on the initial conditions, the azimuthally sensitive femtoscopy of pions was performed with respect to the first-order event plane in $\mathrm{Cu}+\mathrm{Au}$ and $\mathrm{Au}+\mathrm{Au}$ collisions at $\sqrt{s_{N N}}=200 \mathrm{GeV}$.
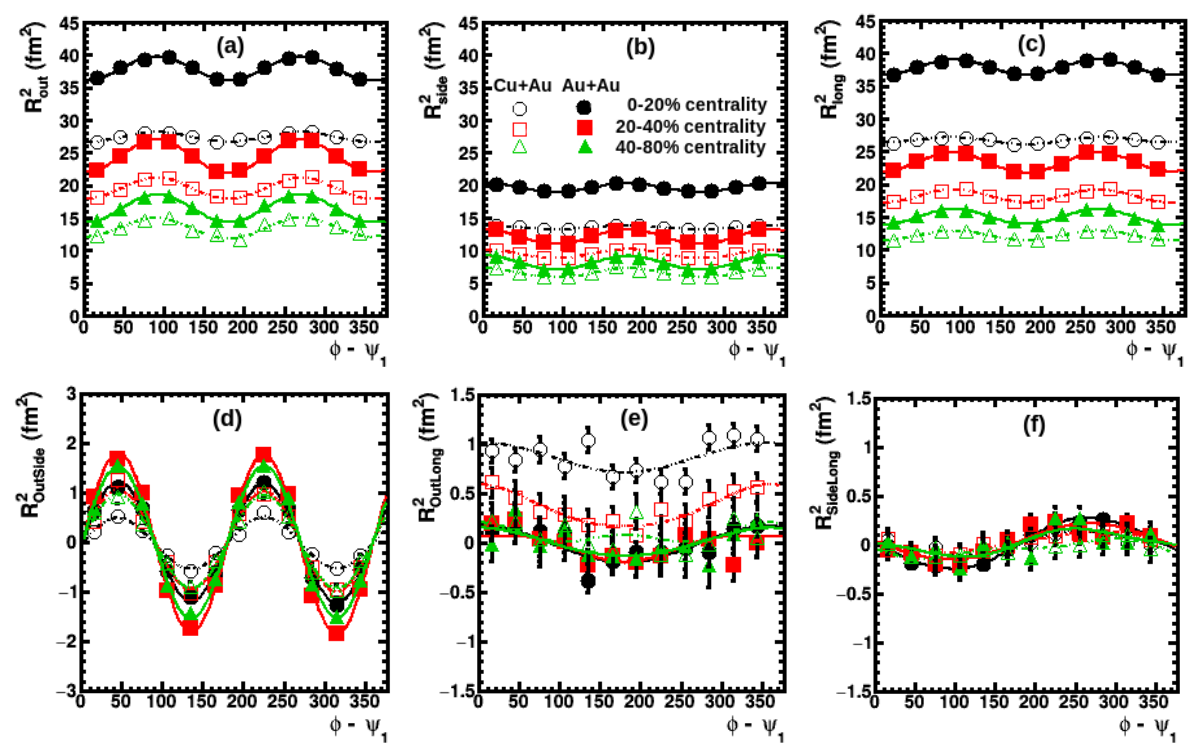

Figure 3. The azimuthal angle dependence of the extracted squared femtoscopic radii for $\mathrm{Au}+\mathrm{Au}$ (solid symbols) and $\mathrm{Cu}+\mathrm{Au}$ (open symbols) at $\sqrt{s_{N N}}=200 \mathrm{GeV}$ for $0.15<k_{T}(\mathrm{GeV} / \mathrm{c})<0.35$. Black circles, red squares and green triangles denote $0-20 \%, 20-40 \%$ and $40-80 \%$ centrality, respectively 
For both of the collision systems the three-dimensional correlation functions were constructed using the Bertsch-Pratt parameterization. These correlation functions were fitted using Eq. 3b. Fig. 3 shows the dependence of the squared radii of pion-emitting source on the difference between the pion pair emission angle, $\phi$, and the reaction plane angle, $\Psi_{1}$, for $\mathrm{Au}+\mathrm{Au}$ (solid symbols) and $\mathrm{Cu}+\mathrm{Au}$ (open symbols) collisions. It should be noted that in the UrQMD model, the angle of the reaction plane is zero. The extracted radii show oscillating dependence on the angle with respect to the first-order event plane. Radii for $\mathrm{Au}+\mathrm{Au}$ collisions are systematically larger than those for $\mathrm{Cu}+\mathrm{Au}$ at the same centrality and pair transverse momentum. The oscillations of the cross-components provide an indication of the tilted source. In order to extract the source tilt, the azimuthal angle dependence of the radii was fitted with equations that corresponds to the Fourier coefficients of the measured radii [15]:

$$
R_{\mu}^{2}(\phi)=R_{\mu, 0}^{2}+2 R_{\mu, 1}^{2} \cos (\phi)+2 R_{\mu, 2}^{2} \cos (2 \phi)
$$

for $\mu=$ out, side, long, out-long fit components, and,

$$
R_{\mu}^{2}(\phi)=R_{\mu, 0}^{2}+2 R_{\mu, 1}^{2} \sin (\phi)+2 R_{\mu, 2}^{2} \sin (2 \phi),
$$

for $\mu=$ out-side, side-long fit components.

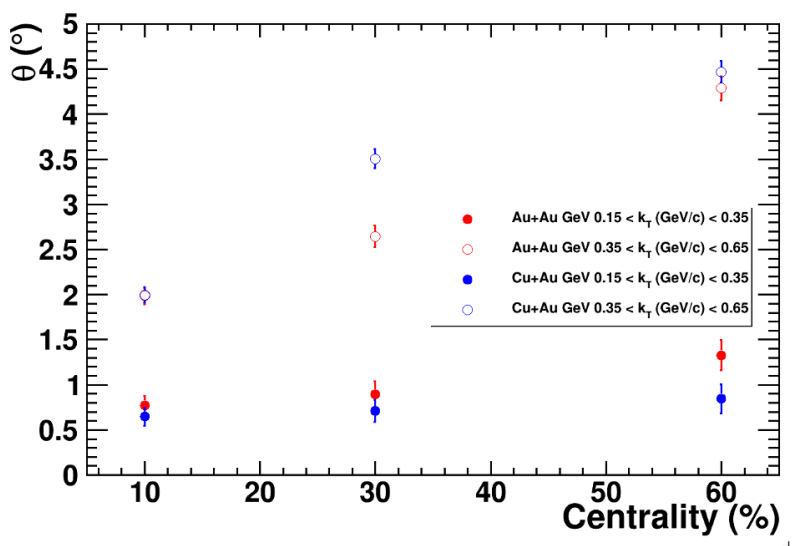

Figure 4. The tilt dependence on the $k_{T}$ and centrality for two collision systems: blue markers - $\mathrm{Cu}+\mathrm{Au}$, red markers - $\mathrm{Au}+\mathrm{Au}$; filled markers $-0.15<k_{T}(\mathrm{GeV} / \mathrm{c})<0.35$, hollow $-0.35<k_{T}(\mathrm{GeV} / \mathrm{c})<0.65$

$$
\theta_{s}=\frac{1}{2} \tan ^{-1}\left(\frac{-4 R_{s l, 1}^{2}}{R_{l, 0}^{2}-R_{s, 0}^{2}+2 R_{s, 2}^{2}}\right)
$$

The tilt angle was calculated using Eq. (6) [7], where $R_{\mu, n}^{2}$ corresponds to the components of the fit functions Eq.(4-5). Fig. 4 shows the dependence of the tilt angle on $k_{T}$ and centrality for the both $\mathrm{Au}+\mathrm{Au}$ and $\mathrm{Cu}+\mathrm{Au}$ systems. The tile angle, $\theta_{s}$ increases with increasing $k_{T}$ and centrality (impact parameter). The $\theta_{s}$ shows a weak (if any) dependence on the collision system. 


\section{Conclusions}

The femtoscopic analysis of small $\left(\mathrm{d}+\mathrm{Au}\right.$ and $\left.{ }^{3} \mathrm{He}+\mathrm{Au}\right)$ and large $(\mathrm{Cu}+\mathrm{Au}$ and $\mathrm{Au}+\mathrm{Au})$ systems at $\sqrt{s_{N N}}=200 \mathrm{GeV}$ has been performed using the data obtained from the UrQMD model.

One-dimensional femtoscopic like-sign pion analysis in the $\mathrm{d}+\mathrm{Au}$ and ${ }^{3} \mathrm{He}+\mathrm{Au}$ systems for similar charged particle multiplicities, $N_{c h}$, and pair transverse momentum, $k_{T}$, have demonstrated the decrease of radii with increasing $k_{T}$ which can be explained by the radial flow. The femtoscopic radii for the $\mathrm{d}+\mathrm{Au}$ and ${ }^{3} \mathrm{He}+\mathrm{Au}$ collisions are consistent within uncertainties for $N_{c h}<20$ and are generally larger for the ${ }^{3} \mathrm{He}+\mathrm{Au}$ collisions for $N_{c h}>20$ as compared to those for the $\mathrm{d}+\mathrm{Au}$ collisions measured for the same $N_{c h}$. It means that the initial geometry of different collision systems cannot be constrained by selecting similar $N_{c h}$.

The azimuthally-differential femtoscopic analysis of charged pions in the $\mathrm{Au}+\mathrm{Au}$ and $\mathrm{Cu}+\mathrm{Au}$ collisions has shown oscillating nature of all 6 components with respect to the firstorder event plane. The fit to these oscillations has allowed one to extract the tilt of the emitting source which was found to be non-zero at $\sqrt{s_{N N}}=200 \mathrm{GeV}$.

Acknowledgments. The reported study was funded by RFBR according to the research project No. 16-02-01119a, by the Ministry of Science and Education of the Russian Federation, grant N 3.3380.2017/4.6 and by the National Research Nuclear University MEPhI in the framework of the Russian Academic Excellence Project (contract No. 02.a03.21.0005, 27.08.2013).

\section{References}

[1] M. Lisa, S. Pratt, R. Soltz, U. Wiedemann Ann. Rev. Nucl. Part. Sci. 55, 55 (2005)

[2] H. Peterson, et al., Phys. Rev. C 78, 18 (2008)

[3] H. Peterson, et al., arXiv:0805.0567 (2008)

[4] M. Bleicher, et al., J. Phys. G 25, 66 (1999)

[5] S. A. Bass, et al., Prog. Part. Nucl. Phys. 41, 129 (1998)

[6] M. A. Lisa, et al., Phys. Lett. B 496, 8 (2000)

[7] M. A. Lisa, U. Heinz, U. A. Wiedemann, Phys. Lett. B 489, 4 (2000)

[8] S. Pratt, Phys. Rev. Lett. 53, 3 (1984)

[9] G. Bretsch, Nucl. Phys. A 489, 6 (1989)

[10] S. Pratt, Phys. Rev. D 33, 14 (1986)

[11] G. Bertsch, M. Gong, M. Tohyama, Phys. Rev. C 37, 5 (1988)

[12] A. N. Makhlin and Y. M. Sinyukov, Z. Phys. C 39, 13 (1988)

[13] S. V. Akkelin, Y. M. Sinyukov, Phys. Lett. B 382, 7 (1995)

[14] B. Abelev et al., Phys. Lett. B 739, 139-151 (2014)

[15] L. Adamzcyk, Phys. Rev. C 92, 27 (2015) 